\title{
Development and Validation of a Chinese Translation of Gambling Expectance Scale through Smallest Space Analysis*
}

\author{
Wen Xue \\ Centre for Studies of Hong Kong, Macao and Pearl River Delta \\ Sun Yat-sen University \\ Guangzhou, China
}

\author{
Zhonglu Zeng \\ Gaming Teaching and Research Centre \\ Macao Polytechnic Institute \\ Macao, China
}

\author{
Zuyun Liu \\ Centre for Studies of Hong Kong, Macao and Pearl River \\ Delta \\ Sun Yat-sen University \\ Guangzhou, China
}

\begin{abstract}
A sample of 312 individuals (residents and tourists) in Macao was investigated by completing a survey assessing their gambling expectancy and the gambling intention. The Smallest Space Analysis (SSA) was adopted as the confirmatory factor analysis (CFA) to validate the Chinese version of gambling expectancy scale's sub-dimensions generated by the exploratory factor analysis (EFA). 10 items to judge people's gambling intention were adopted as validate variables to investigate their associations with the gambling expectancy. In the configuration produced by the Gambling Expectancy Questionnaire (GEQ), results indicated that the 3dimension solution of the scale was valid for the Chinese sample. The SSA results revealed that all the gambling intention variables were located in the same region between potential gains and self or parents' evaluation, indicating that people believe gambling could bring the potential gains and higher self/evaluation were more likely to commit gambling. Compared with the gamblers, non-gamblers scored higher on punishment dimension, but lower than potential gain dimension. Chinese gamblers were found gambling for reach better social status, which in line with Chinese cultural background.
\end{abstract}

Keywords-gambling expectancy; gambling intention; the smallest space analysis; non-gambler

\section{INTRODUCTION}

Gambling is found across many cultures, but, it reflects specific expressions various in different culture [1-2]. According to the report from Macao University (2017), one out of ten gamblers is a problem gambler in Macao. Problem gambling has caused a lot of serious social problems in Macao, including social isolation, divorcement, and fraud. Currently, the problem gambling rate was reported much

*This project is sponsored by China Postdoctoral Research Funding, the Project number is 2017M622842 higher in the Chinese sample than found in Caucasian cultures [3-5]. However, compared to the majority of the gambling research conducted in northern America, Europe, and Australia [6-8], its counterpart in Asian sample is still comparatively few. Therefore, it worth to develop a better understanding of psychosocial factors that can explain the development and maintenance of problem gambling in Asian countries, so that effective prevention and intervention programs can be put together.

Behaviorists believe that people's attitudes or motives can affect their decision making on a risk or hazard [9] Decision making under risk and uncertainty has been one of the most active and interdisciplinary research topics in judgment and decision making. Risk perception is the subjective judgment that people make about the characteristics and severity of a risk. Our understandings of risk perception have come from geography, sociology, political science, and also psychology. The concept of risk perception has been applied in many fields, such as gambling, health care and climate change, to predict policy preferences and behaviors. A recent systematic review of risk perception in gambling that examined existing research of decision making and gambling behavior found that users' expectations of potential outcomes are important to moderate gambling behaviors [10]. However, despite an extensive focus on cognitive biases associated with problem gambling, a paucity of research about gamblers' perceptions of potential harms and risk related to gambling appeals more studies to be conducted in this area. To fix this research gap, the current research is trying to develop a gambling perception scale especially predict people's gambling expectance under a Chinese cultural context. 


\section{A. The Smallest Space Analysis}

To test the validity of the Chinese gambling expectancy scale, we adopted the smallest space analysis by using its plot function to better understand the measurement, and also better presentation of the relations between gambling intention and gambling expectancy. The SSA is a multidimensional scaling technique that was firstly introduced and developed by Guttman [11], which maps the location of each item in a multidimensional space. It treats each observed variable as a point in this multidimensional space, so that the higher the correlation between two variables, the closer they would be presented in the space [11]. The location of each item is determined by assessing the similarity or dissimilarity to all of the other items. SSA can produce two indicators to evaluate the configuration quality [12]: the coefficient of alienation and the separation index, which reflects how it fit between the spatial configuration and the data (values of this index range from 0 (best) to 1 (worst), while the separation index examines the fit between the spatial solution found in the confirmatory SSA and the a priori spatial hypothesis derived from the mapping sentence, which ranges from 0 (worst) to 1 (best) [12].

\section{B. Current Research}

The aim of current research is develop and validate a Chinese version of gambling expectance scale in line with Chinese social background and context. We hypothesised that The Chinese version of the gambling expectance scale can well predict the gambler's gambling intention in future. We also further predicted that there should be significant difference of gambling risk perception between the gambler group and non-gambler group. We also aimed to address methodological shortcomings of existing measures.

\section{METHOD}

\section{A. Participants and Procedure}

In this study, Questionnaires of the Simplified Chinese version Scales were administered in early January and February, 2018. Of 350 questionnaires handed out in Macao, 312 usable questionnaires were collected for the residents in Macao and the tourists from China. The investigating location includes famous casinos in Macao (e.g., Venetian, Parisian \& Galaxy), and famous tourism points (e.g., Ruin of St. Paul). The survey assessed people's gambling perception through the Chinese version of the Gambling Expectancy Questionnaire (GEQ) [13]. Participant letter was shown at the first page of the survey to the participants to explain the research background and aim of the project. All responses were anonymous. Participants involved in this investigation were aged from $18-73$ years, with $46.3 \%$ females and $53.7 \%$ males. Only $34.6 \%$ of the participants received college education level or above, and $67.7 \%$ of them currently have a full-time/part-time job.

\section{B. Measures}

The survey consists of the following measures:
- Participant's demographic information, such as age, education level, and employment, etc.

- The second section is the Gambling Expectancy Questionnaire [13], which is to assess negative or positive outcomes perceived by participants if they attend to gamble.

- Gambling intention: 10-item gambling intention scale developed by Moore \& Ohtsuka [14], asking how likely the participants would like to attend to the following games in future, such as (1) Poker machine (2) Lottery; (3) Horses, with all assessed on a 5-point scale from (1) not likely to (5) very likely. The cronbach's of this scale is 0.89 .

\section{RESULT}

\section{A. Factor Analysis for the GEQ}

The cronbach's alpha for the Chinese version of GEQ before deleting any items was 0.92 , indicating a good level of internal consistency [15].

TABLE I. FACTOR PATTERN MATRIX FOR THE EFA

\begin{tabular}{|c|c|c|c|}
\hline & \multicolumn{3}{|c|}{ Pattern Matrix ${ }^{a}$} \\
\hline & \multicolumn{3}{|c|}{ Factor } \\
\hline & 1 & 2 & 3 \\
\hline Better life & 0.75 & & \\
\hline Get Money & 0.70 & & \\
\hline New experiences & 0.69 & & \\
\hline Outlook & 0.64 & & \\
\hline Money problem & 0.64 & & \\
\hline Winner & 0.58 & & \\
\hline Top of the world & 0.57 & & \\
\hline Parents happy & & 0.64 & \\
\hline Proud & & 0.57 & \\
\hline Free & & 0.55 & \\
\hline Parents proud & & 0.54 & \\
\hline Self-esteem & 0.50 & 0.52 & \\
\hline Beat up & & & 0.78 \\
\hline Law trouble & & & 0.72 \\
\hline Get caught & & & 0.69 \\
\hline Dangerous & & & 0.45 \\
\hline
\end{tabular}

a. Note. Extraction Method: Principal Axis Factoring. a. 3 factors extracted. 5 iterations required Items load less than 0.45 were not reported

An EFA was conducted using principal-axis factoring in SPSS (Version 20). To determine the number of factors to retain, we applied both Kaiser's eigenvalues-greater-thanone rule [16], and scree plot to the unrotated solution. In the initial report of EFA for the GEQ scale, 4 components have eigenvalues more than 1 . The $\mathrm{KMO}$ value is $0.94, \mathrm{p}<0.001$, and total explained $64.7 \%$ variance. However, the scree plot indicated that a 3 -factor solution. Solutions between 2 and 4 factors were then subjected to direct oblimin rotations $(\Delta=0)$ and assessed for interpretability. A 3-factor solution with 3 meaningful factors was judged to be most interpretable in "Table I". This solution accounted for $59.7 \%$ of the total variance in the GEQ scale items. Specifically, factor 1 consists of all 3 items in material gain dimension in the original GEQ scale, and the other items are about other positive gains, e.g. the social status they can obtain "top of the world", and "new experience". So this factor should be 
named as potential gains. Factor 2 is highly related to the self and parents evaluation, while factor 3 contains all estimated punishments after gambling, which can be considered as the negative consequence of gambling.

\section{B. Confirmatory Smallest Space Analysis (SSA) for the GEQ Scale}

To test the validation of the EFA solution, a confirmatory smallest space analysis was conducted was performed based on the prediction about the dimensional structure of GEQ scale in the SSA space through the HUDAP software. The coefficient of alienation for the three-dimension solution is 0.07, which suggests an acceptable level according to Amar [17]. The SSA plot is shown in figure 1. The obtained pattern of the dimensions generated by the EFA in the GEQ scale reflects a clear polar structure with three partitions (separation index $=0.90$ ). The perspective facet divides the map into three regions: The left region includes items regarding whether the respondents holding a strong belief of negative outcomes if they do gambling, whereas items located in the upper right area assess the respondents' perceptions regarding the possible gains within gambling, and the bottom right region explains how they evaluate their parents and themselves.

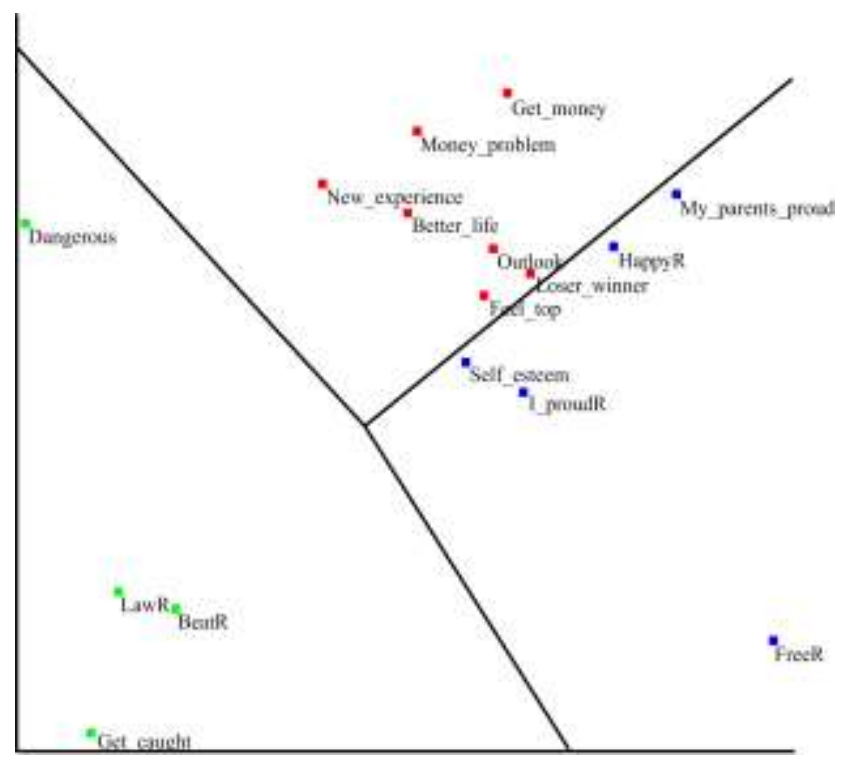

Fig. 1. The smallest space analysis plot for the GRQ scale.

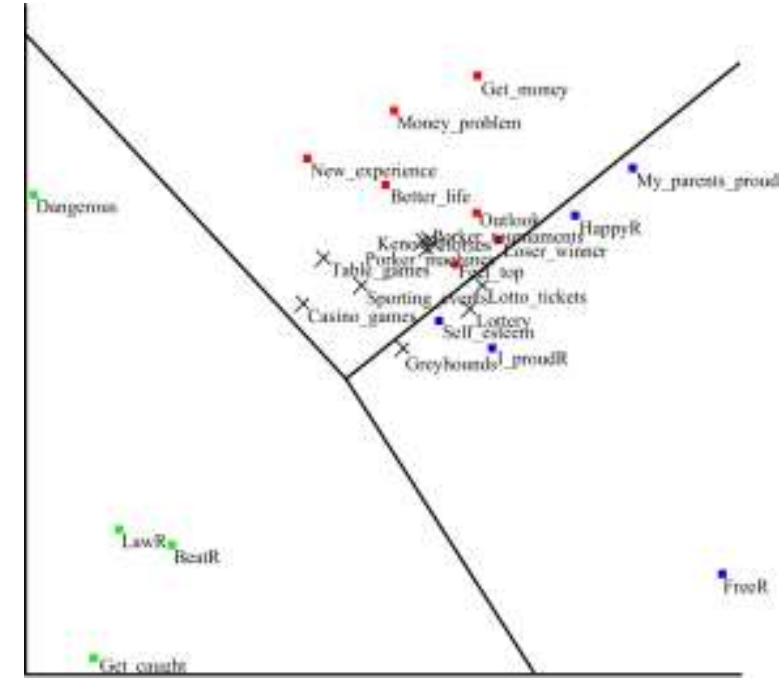

Fig. 2. The distribution of gambling intention in the GRQ plot.

\section{The Relations between Gambling Intention and Gambling Expectance}

The 10 items in the gambling intention were submitted in the analysis as external variables and added to the spatial configuration of GRQ through the HUDAP software. Their locations are depicted in the plot generated by GRQ (see Figure 2). Our hypothesis was supported by that all 10 intention items are in the area between the potential gain partition and self and parents' evaluation partition in the figure, while none of them located in the negative consequence part. Most of gambling intention items are highly related to item "gambling would make me feel like I'm on the top of the world", "gambling would make me a total winner", and item "gambling would make me have a very positive outlook on life".

\section{Different Risk Perception between Gamblers and Non- gamblers}

A correlation coefficient analysis suggested that all those dimensions derived from the GEQ scale are good predictors for individual's gambling intention in next 12 months, with the highest effect size of dimension one, potential gains $(\mathrm{r}=0.84, \mathrm{p}<0.001)$ (See table 2$)$. The participant's identity, gambler or not is also highly related to their gambling expectance, with an effect size from 0.29 to 0.33 of these three GEQ dimensions at a significant level of $\mathrm{p}<0.001$. To further examine the risk perception difference between gamblers and non-gamblers, an independent T-test was also conducted. The gamblers reported higher score potential gain dimension $(\mathrm{M}=2.60, \mathrm{SD}=0.77$, and self/parents evaluation $(\mathrm{M}=2.66, \mathrm{SD}=0.63)$, but lower in punishment dimension $(\mathrm{M}=2.74, \mathrm{SD}=0.88)$; while non-gamblers reported lower score on these two dimension $(\mathrm{M}=2.05, \mathrm{SD}=0.79 ; \mathrm{M}=2.23$, $\mathrm{SD}=0.76)$, but higher on the punishment dimension $(\mathrm{M}=3.34$, $\mathrm{SD}=0.89$ ). The independent $\mathrm{T}$-test result of the first dimension is $\mathrm{t} 1$ (253) $=5.61, \mathrm{p}<0.001$; for the second dimension is $\mathrm{t} 2=4.84, \mathrm{p}<0.001$; and for the punishment dimension is $\mathrm{t} 3(253)=5.38, \mathrm{p}<0.001$. 
TABLE II. ZERO-ORDER CORRELATIONS AND DESCRIPTIVE STATISTICS FOR ALL VARIABLES

\begin{tabular}{|c|c|c|c|c|c|c|c|}
\hline $\begin{array}{c}\text { Variabl } \\
\text { e }\end{array}$ & 1 & 2 & 3 & 4 & 5 & 6 & 7 \\
\hline $\begin{array}{l}\text { Gambler } \\
\text { s or Not }\end{array}$ & & & & & & & \\
\hline Gender & $0.19 * *$ & & & & & & \\
\hline Employ & $\begin{array}{ll}0 & .21 * \\
* & \end{array}$ & 0.002 & & & & & \\
\hline $\begin{array}{l}\text { Educatio } \\
\mathrm{n}\end{array}$ & -0.04 & 0.11 & 0.08 & & & & \\
\hline GEQ1 & $\begin{array}{l}- \\
0.33 * * \\
*\end{array}$ & $\begin{array}{l}- \\
0.26 * * \\
*\end{array}$ & $\begin{array}{l}- \\
0.04\end{array}$ & $\begin{array}{l}- \\
0.05\end{array}$ & & & \\
\hline GEQ2 & $\begin{array}{l}- \\
0.29 * * \\
*\end{array}$ & $\begin{array}{l}- \\
0.22 * * \\
*\end{array}$ & $\begin{array}{l}- \\
0.02\end{array}$ & $\begin{array}{l}- \\
0.06\end{array}$ & $\begin{array}{l}0.84 \\
* * *\end{array}$ & & \\
\hline GEQ3 & $\begin{array}{l}0.32 * * \\
*\end{array}$ & $0.13^{*}$ & 0.04 & 0.02 & $\begin{array}{l}- \\
0.50 \\
* * *\end{array}$ & $\begin{array}{l}- \\
0.47 \\
* * *\end{array}$ & \\
\hline $\begin{array}{l}\text { Gamblin } \\
\mathrm{g} \\
\text { intention }\end{array}$ & $\begin{array}{l}- \\
0.28 * * \\
*\end{array}$ & $\begin{array}{l}- \\
0.17 * *\end{array}$ & 0.01 & $\begin{array}{l}- \\
0.04\end{array}$ & $\begin{array}{l}0.52 \\
* * *\end{array}$ & $\begin{array}{l}0.50 \\
* * *\end{array}$ & $\begin{array}{l}- \\
0.28 * * \\
*\end{array}$ \\
\hline
\end{tabular}

b. $* \mathrm{p}<0.05, * * \mathrm{p}<0.01, * * * \mathrm{p}<0.001$. For gamblers or not: $1=$ Gamblers, $2=$ Non-gamblers, $0=$ Not disclose/don't know (59 respondents prefer not disclose their identity). To better understand the context, dimension 3 of GEQ is reverse coded.

\section{CONCLUSION}

In this study, we aimed to develop and validate a Chinese version of the gambling expectance scale, and also test the risk perception difference between gambles and nongamblers. The results supported our hypotheses. In the confirmatory analysis, we found that all the gambling intention variables were located in the same region between potential gains and self or parents' evaluation partition, which suggested that people who believe gambling could bring the potential gains and higher self/evaluation were more likely to commit gambling. Compared with the gamblers, non-gamblers scored higher on punishment dimension, but lower than potential gain dimension. This result indicated non-gamblers are concerned about the negative consequence more than gamblers, and hold less positive attitudes towards the rewards of gambling behaviour. Last, Chinese gamblers were found that their main reason for gambling is to achieve better social status, which in line with Chinese cultural background.

\section{REFERENCES}

[1] Raylu, N., \& Oei, T. P. (2004). The Gambling Related Cognitions Scale (GRCS): Development, confirmatory factor validation and psychometric properties. Addiction, 99(6), 757-769.

[2] Custer, R., \& Milt, H. (1985). When luck runs out: Help for compulsive gamblers. NewYork: Facts on File Publications.

[3] Wardle, M. C., Gonzalez, R., Bechara, A., \& Martin-Thormeyer, E. M. (2010). Lowa Gambling Task performance and emotional distress interact to predict risky sexual behavior in individuals with dual substance and HIV diagnoses. Journal of clinical and experimental neuropsychology, 32(10), 1110-1121.

[4] Fong, D. K., \& Ozorio, B. (2005). Gambling participation and prevalence estimates of pathological gambling in a far-east gambling city: Macao. UNLV Gaming Research \& Review Journal, 9(2), 15-28.

[5] Shaffer, H. J., Hall, M. N., \& Vander Bilt, J. (1999). Estimating the prevalence of disordered gambling behavior in the United States and
Canada: a research synthesis. American journal of public health, 89(9), 1369-1376.

[6] Petry, N. M. (2005). Stages of change in treatment-seeking pathological gamblers. Journal of consulting and clinical psychology, 73(2), 312-322.

[7] Raylu, N., \& Oei, T. P. (2002). Pathological gambling: A comprehensive review. Clinical psychology review, 22(7), 1009-1061.

[8] Scherrer, J. F., Slutske, W. S., Xian, H., Waterman, B., Shah, K. R., \& Volberg, R., et al. (2007). Factors associated with pathological gambling at 10-year follow-up in a national sample of middle-aged men. Addiction, 102(6), 970-978.

[9] Loewenstein, G. F., Weber, E. U., Hsee, C. K., \& Welch, N. (2001) Risk as feelings. Psychological Bulletin, 127(2), 267-286. doi: 10.1037//0033-2909.127.2.267

[10] Spurrier, M., \& Blaszczynski, A. (2014). Risk perception in gambling: a systematic review. Journal of Gambling Studies, 30(2), 253-276.

[11] Guttman, L. (1968). A generalized nonmetric technique for finding the smallest coordinate space for a configuration of points. Psychometrika, 33, 469-506.

[12] Shye, S., Elizur, D., \& Hoffman, M. (1994). Introduction to facet theory. Thousand Oaks, CA: Sage.

[13] Wickwire, E. M., Whelan, J. P., \& Meyers, A. W. (2010). Outcome expectancies and gambling behavior among urban adolescents. Psychology of Addictive Behaviors, 24(1), 75-88.

[14] Moore, S. M., \& Ohtsuka, K. (1997). Gambling activities of young australians: developing a model of behaviour. Journal of Gambling Studies, 13(3), 207.

[15] Devellis, R. F. (1991). Scale development:, theory and applications. , 26.

[16] Kaiser, H. F. (1960). The application of electronic computers to factor analysis. Educational and Psychological Measurement, 20, 141-151.

Amar, R. (2005). Hudap Manual (Third ed.): The Hebrew University of Jerusalem Computation Authority. 